\title{
Current Exchanges for Reducible Higher Spin Modes on AdS
}

\author{
A. Fotopoulos, M. Tsulaia
}

\begin{abstract}
We show how to decompose a Lagrangian for reducible massless bosonic Higher Spin modes into the ones describing irreducible (Fronsdal) Higher Spin modes on a $\mathcal{D}$ dimensional $A d S$ space. Using this decomposition we construct a new nonabelian cubic interaction vertex for reducible higher spin modes and two scalars on $A d S$ from the already known vertex which involves irreducible (Fronsdal) modes.
\end{abstract}

Keywords: gauge symmetry, AdS-CFT correspondence, string field theory.

Higher Spin gauge theories (see [1]-[2] for recent reviews) are usually formulated either in frame-like [3]-[4] or metric-like [5]-[20] approaches.

Recently several interesting cubic vertices have been constructed in the metric-like approach $[8,9$, $10,11]$. Bearing in mind a possible application of the reducible Higher Spin modes (described by the socalled "triplet" [20]) for String Theory [21]-[22] and for AdS/CFT correspondence [23], we consider the problem of cubic interaction of a triplet on an AdS space. In particular we study the cubic interaction of a triplet with two scalar fields.

The main result of this paper is twofold. Firstly, we show that the procedure derived in [17] for decomposing the free Lagrangian for reducible massless bosonic Higher Spin modes in a flat space time also works for an arbitrary dimensional AdS space. The second and more important result is that after this decomposition one can use the cubic vertex ${ }^{1}$ of [16], which describes an interaction of irreducible (Fronsdal) Higher Spin modes with two scalars, to obtain an interaction vertex for reducible Higher Spin modes with two scalars. Obviously this technique can be applied not only for the particular vertex given in [16], but for constructing of more complicated interaction vertices in AdS following the method given in [14]. The advantage of this approach is that the construction of interaction vertexes for triplets in AdS is often technically complicated, due to repeated commutators between covariant derivatives. The double tracelessness condition for irreducible Higher Spin modes makes the problem at hand considerably simpler.

Let us start from a free Lagrangian describing the propagation of reducible massless Higher Spin modes on a $\mathcal{D}$ dimensional $\mathrm{AdS}$ space. It contains a field $\varphi_{\mu_{1}, \ldots, \mu_{s}}(x)$ of rank $s$, a field $C_{\mu_{1}, \ldots, \mu_{s-1}}(x)$ of rank $s-1$ and a field $D_{\mu_{1}, \ldots, \mu_{s-2}}(x)$ of rank $s-2$, and has the form [13] (see also [2] for details of the construction),

$$
\begin{aligned}
\mathcal{L}= & -\frac{1}{2}\left(\nabla_{\mu} \varphi\right)^{2}+s \nabla \cdot \varphi C+s(s-1) \nabla \cdot C D+ \\
& \frac{s(s-1)}{2}\left(\nabla_{\mu} D\right)^{2}-\frac{s}{2} C^{2}+\frac{s(s-1)}{2 L^{2}}\left(\varphi^{\prime}\right)^{2}- \\
& \frac{s(s-1)(s-2)(s-3)}{2 L^{2}}\left(D^{\prime}\right)^{2}- \\
& \frac{4 s(s-1)}{L^{2}} D \varphi^{\prime}- \\
& \frac{1}{2 L^{2}}[(s-2)(\mathcal{D}+s-3)-s] \varphi^{2}+ \\
& \frac{s(s-1)}{2 L^{2}}[s(\mathcal{D}+s-2)+6] D^{2}
\end{aligned}
$$

The symbol $\nabla$. means divergence, while $\nabla$ is the symmetrized action of $\nabla_{\mu}$ on a tensor. The symbol' means that we take the trace of a field. Multiplication of a tensor by the metric $g$ implies symmetrized multiplication, i.e., if $A$ is a vector $A_{\mu}$ we have $g A=g_{(\mu \nu} A_{\rho)}=g_{\mu \nu} A_{\rho}+g_{\mu \rho} A_{\nu}+g_{\nu \rho} A_{\mu}$. This Lagrangian is invariant under gauge transformations with parameter $\Lambda_{\mu_{1}, \ldots, \mu_{s-1}}(x)$

$$
\begin{aligned}
\delta \varphi & =\nabla \Lambda, \\
\delta C & =\square \Lambda+\frac{(s-1)(3-s-\mathcal{D})}{L^{2}} \Lambda+\frac{2}{L^{2}} g \Lambda^{\prime} \\
\delta D & =\nabla \cdot \Lambda .
\end{aligned}
$$

Let us note that the field $C(x)$ has no kinetic term and can be eliminated via its own equations of motion to obtain

$$
\begin{aligned}
\mathcal{L}= & -\frac{1}{2}\left(\nabla_{\mu} \varphi\right)^{2}+\frac{s}{2}(\nabla \cdot \varphi)^{2}+ \\
& s(s-1) \nabla \cdot \nabla \cdot \varphi D+s(s-1)\left(\nabla_{\mu} D\right)^{2}+
\end{aligned}
$$

Talk given at the XIXth International Colloquium on Integrable Systems and Quantum Symmetries, Prague, Czech Republic, June $17-19,2010$

${ }^{1}$ Let us point out that the method given in [14] describes the construction of nonabelian cubic interaction vertices, see also [16] for some explicit nonabelian examples. A particular example of an abelian vertex given in [15] is in some sense a "degenerate" solution of the method, where however the abelian property is maintained in a nontrivial way, due to the structure of the ghost terms. 


$$
\begin{aligned}
& \frac{s(s-1)(s-2)}{2}(\nabla \cdot D)^{2}+ \\
& \frac{s(s-1)}{2 L^{2}}\left(\varphi^{\prime}\right)^{2}- \\
& \frac{s(s-1)(s-2)(s-3)}{2 L^{2}}\left(D^{\prime}\right)^{2}- \\
& \frac{4 s(s-1)}{L^{2}} D \varphi^{\prime}- \\
& \frac{1}{2 L^{2}}[(s-2)(\mathcal{D}+s-3)-s] \varphi^{2}+ \\
& \frac{s(s-1)}{2 L^{2}}[s(\mathcal{D}+s-2)+6] D^{2} .
\end{aligned}
$$

Now we would like to decompose this Lagrangian in terms of irreducible (Fronsdal) [6] modes, following the procedure given in [17] for a Minkowski space.

Let us start with the simplest example of a $s=2$ triplet which contains fields $\varphi_{\mu \nu}(x), C_{\mu}(x)$ and $D(x)$. Let us make the ansatz

$$
\varphi_{\mu \nu}=\Psi_{\mu \nu}+\frac{1}{\mathcal{D}-2} g_{\mu \nu} \Psi, \quad \varphi^{\prime}-2 D=\Psi .
$$

Inserting these expressions back to the Lagrangian (3), for $s=2$ one obtains

$$
\begin{aligned}
\mathcal{L}= & -\frac{1}{2}\left(\nabla_{\mu} \Psi_{\rho \sigma}\right)^{2}+\left(\nabla_{\nu} \Psi_{\mu}^{\nu}\right)^{2}+\Psi^{\prime} \nabla_{\mu} \partial_{\nu} \Psi^{\mu \nu}+ \\
& \frac{1}{2}\left(\nabla_{\mu} \Psi^{\prime}\right)^{2}-\frac{1}{2(\mathcal{D}-2)}\left(\nabla_{\mu} \Psi\right)^{2}+ \\
& \frac{1}{L^{2}}\left(\Psi_{\mu \nu}\right)^{2}+\frac{\mathcal{D}-3}{L^{2}(\mathcal{D}-2)}(\Psi)^{2}+\frac{\mathcal{D}-3}{2 L^{2}}\left(\Psi^{\prime}\right)^{2}
\end{aligned}
$$

Therefore, the initial Lagrangian (3) has been decomposed into a sum of two Fronsdal Lagrangians for $s=2$ field $\Psi_{\mu \nu}$ with the gauge transformation law $\delta \Psi_{\mu \nu}=\nabla_{\mu} \Lambda_{\nu}+\nabla_{\mu} \Lambda_{\nu}$ and a gauge invariant scalar $\Psi$.

Let us describe this procedure for the spin 4 triplet, since in this case both a constraint on the parameter of gauge transformations and an off-shell constraint on the gauge field arise. Let us use the substitution [17]

$$
\begin{aligned}
\varphi^{(4)}= & \Psi^{(4)}+\frac{1}{\mathcal{D}+2} g \Psi^{(2)}+\frac{1}{\mathcal{D}(\mathcal{D}-2)}(g)^{2} \Psi^{(0)} \\
D= & \frac{1}{2}\left[\Psi^{\prime(4)}+\frac{2}{\mathcal{D}+2} \Psi^{(2)}+\frac{1}{\mathcal{D}+2} g \Psi^{\prime(2)}+\right. \\
& \left.\frac{2}{\mathcal{D}(\mathcal{D}-2)} g \Psi^{(0)}\right]
\end{aligned}
$$

The field $\Psi^{(4)}$ is doubly traceless and transforms under the gauge transformations as

$$
\delta \Psi^{(4)}=\nabla \tilde{\Lambda}, \quad \tilde{\Lambda}=\Lambda-\frac{1}{\mathcal{D}+2} \eta \Lambda^{\prime}
$$

Inserting these expressions into the Lagrangian (3), one can see again that it decomposes into the sum of the Fronsdal modes with spins 4,2 and 0, described by the fields $\Psi^{(4)}, \Psi^{(2)}$ and $\Psi^{(0)}$.

One can further generalize this procedure for an arbitrary spin. In particular, take

$$
\begin{aligned}
\varphi= & \sum_{k=0}^{\left[\frac{s}{2}\right]} \tilde{\rho}_{k}(\mathcal{D}, s)(g)^{k} \Psi^{(s-2 k)} \\
D= & \frac{1}{2} \sum_{k=0}^{\left[\frac{s}{2}\right]-1} \tilde{\rho}_{k}(\mathcal{D}, s)(g)^{k} \Psi^{\prime}(s-2 k)+ \\
& \sum_{k=1}^{\left[\frac{s}{2}\right]} \tilde{\rho}_{k}(\mathcal{D}, s)(g)^{k-1} \Psi^{(s-2 k)} .
\end{aligned}
$$

and

$$
\tilde{\Lambda}_{s-1-2 k}=\sum_{q=0}^{\left[\frac{s}{2}\right]} \rho_{q}(\mathcal{D}, s-2 k-1)(g)^{q} \Lambda^{[q+k](s-1)}
$$

with

$$
\begin{aligned}
\rho_{q}(\mathcal{D}-2, s) & =\frac{(-1)^{q}(\mathcal{D}+2(s-q-3)) ! !}{(\mathcal{D}+2(s-3)) ! !} \\
\tilde{\rho}_{k}(\mathcal{D}, s) & =\frac{(\mathcal{D}+2(s-2 k-2)) ! !}{(\mathcal{D}+2(s-k-2)) ! !}
\end{aligned}
$$

and $[q+k]$ denotes the number of traces. Finally, one can show that the normalization factor for the propagators for each of individual Fronsdal mode, i.e. the inverse of the prefactor of $\left(\nabla_{\mu} \Psi^{(s-2 k)}\right)^{2}$ terms multiplied by 2 , is

$$
Q(s, k, \mathcal{D})=\frac{2^{k} k !(s-2 k) !}{s ! \tilde{\rho}_{k}(\mathcal{D}, s)}
$$

Now let us build a cubic interaction vertex of a Higher Spin triplet with two scalars on AdS. To this end, let us use the corresponding vertex for an individual Fronsdal mode [16]

$$
\begin{aligned}
\mathcal{L}_{\text {int }}^{00 s}= & \Psi^{(s)} \cdot J_{s}+\left[\frac{s-1}{6 L^{2}}\left[2 s^{2}+(3 \mathcal{D}-4) s-6\right]-\right. \\
& \left.\frac{s-2}{L^{2}}\right] \Psi^{\prime(s)} \cdot J_{s-2}
\end{aligned}
$$

where

$$
\begin{aligned}
J_{s-2 q}^{1 ; 2}= & \sum_{r=0}^{s-2 q} C_{s-2 q}^{r}(-1)^{r}\left(\nabla^{\mu_{1}} \ldots \nabla^{\mu_{r}} \phi_{1}\right) . \\
& \left(\nabla^{\mu_{r+1}} \ldots \nabla^{\mu_{s-2 q}} \phi_{2}\right)
\end{aligned}
$$

Therefore multiplying the interacting vertices (12) with the appropriate factor (11) and adding them to the free Lagrangian (3), one finds the expression for a cubic Lagrangian describing the interaction of reducible Higher Spin modes with two scalars on AdS. Then one can perform a current-current exchange procedure following the lines of $[18,19,17]$. 


\section{Acknowledgement}

It is a pleasure to thank A. P. Isaev, S. O. Krivonos and A. O. Sutulin for valuable discussions. The work of A. F. was supported by an INFN postdoctoral fellowship and partly supported by Italian MIUR-PRIN contract 20075 ATT78. The work of M. T. has been supported by a STFC rolling grant ST/G00062X/1.

\section{References}

[1] Vasiliev, M. A.: Fortsch. Phys. 52, 702 (2004) [arXiv:hep-th/0401177]. Bekaert, X., Cnockaert, S., Iazeolla, C., Vasiliev, M. A.: [arXiv:hepth/0503128]. Sorokin, D.: AIP Conf. Proc. 767, 172 (2005) [arXiv:hep-th/0405069]. Bouatta, N., Compere, G., Sagnotti, A.:

[arXiv:hep-th/0409068]. Bekaert, X., Buchbinder, I. L., Pashnev, A., Tsulaia, M.: Class. Quant. Grav. 21 (2004) S1457 [arXiv:hepth/0312252]. Campoleoni, A.: arXiv:0910.3155, Francia, D.: J. Phys. Conf. Ser. 222, 012002 (2010) [arXiv:1001.3854]. Bekaert, X., Boulanger, N., Sundell, P., [arXiv:1007.0435].

[2] Fotopoulos, A., Tsulaia, M: Int. J. Mod. Phys. A 24, 1 (2009) [arXiv:0805.1346 [hep-th]].

[3] Fradkin, E. S., Vasiliev, M. A.: Nucl. Phys. B 291, 141 (1987), Annals Phys. 177, 63 (1987), Vasiliev, M. A.: Phys. Lett. $B$ 243, 378 (1990), Phys. Lett. B 285, 225 (1992), Phys. Lett. B 567, 139 (2003) [arXiv:hep-th/0304049], arXiv:0707.1085 [hep-th], JHEP 0412 (2004) 046 [arXiv:hep-th/0404124], Skvortsov, E. D., Vasiliev, M. A.: Nucl. Phys. B 756 (2006) 117 [arXiv:hep-th/0601095], Sorokin, D. P., Vasiliev, M. A.: Nucl. Phys. B 809, 110 (2009) [arXiv:0807.0206 [hep-th]],

[4] Bandos, I. A., Lukierski, J., Sorokin, D. P.: Phys. Rev. D 61, 045002 (2000) [arXiv:hepth/9904109]. Vasiliev, M. A.: Phys. Rev. D 66, 066006 (2002) [arXiv:hep-th/0106149].

[5] C. Fronsdal, Phys. Rev. D 18, 3624 (1978), Berends, F. A., Burgers, G. J. H., van Dam, H.: Nucl. Phys. B 271, 429 (1986), Ouvry, S., Stern, J.: Phys. Lett. $B$ 177, 335 (1986), Bengtsson, A. K. H., Bengtsson, I., Brink, L.: Nucl. Phys. B 227, 41 (1983), Nucl. Phys. B 227, 31 (1983), Hussain, F., Thompson, G., Jarvis, P. D.: Phys. Lett. B 216, 139 (1989).

[6] C. Fronsdal, Phys. Rev. D 20, 848 (1979).

[7] Pashnev, A., Tsulaia, M. M.: Mod. Phys. Lett. A 12, 861 (1997) [arXiv:hep-th/9703010]. Buchbinder, I. L., Pashnev, A., Tsulaia, M.: Phys.
Lett. B 523 (2001) 338 [arXiv:hep-th/0109067]. [arXiv:hep-th/0206026]. Buchbinder, I. L., Krykhtin, V. A., Pashnev, A.: Nucl. Phys. B 711, 367 (2005) [arXiv:hep-th/0410215]. Buchbinder, I. L., Krykhtin, V. A., Ryskina, L. L., Takata, H.: Phys. Lett. B 641 (2006) 386 [arXiv:hep-th/0603212]. Buchbinder, I. L., Galajinsky, A. V., Krykhtin, V. A.: [arXiv:hepth/0702161]. Buchbinder, I. L., Krykhtin, V. A., Reshetnyak, A. A.: [arXiv:hep-th/0703049]. Buchbinder, I. L., Krykhtin, V. A., Lavrov, P. M.: Nucl. Phys. B $\mathbf{7 6 2}$ (2007) 344 [arXiv:hep-th/0608005]. Alfaro, J., Cambiaso, M., [arXiv:0809.4298 [hep-th]]. Alkalaev, K. B., Grigoriev, M.: Nucl. Phys. B 835, 197 (2010) [arXiv:0910.2690 ]. Isaev, A. P., Krivonos, S. O., Ogievetsky, O. V.: J. Math. Phys. 49, 073512 (2008) [arXiv:0802.3781 [math-ph]].

[8] Metsaev, R. R.: [ arXiv:hep-th/9810231], Phys. Lett. B 419, 49 (1998) [arXiv:hep-th/9802097], [arXiv:0712.3526 [hep-th]], Nucl. Phys. B 759, 147 (2006) [arXiv:hep-th/0512342],

[9] Bekaert, X., Boulanger, N., Cnockaert, S.: JHEP 0601, 052 (2006)

[arXiv:hep-th/0508048], Boulanger, N., Leclercq, S., Cnockaert, S.: Phys. Rev. D 73 (2006) 065019 [arXiv:hep-th/0509118], Boulanger, N., Leclercq, S.: JHEP 0611 (2006) 034

[arXiv:hep-th/0609221], Boulanger, N., Leclercq, S., Sundell, P.: JHEP 0808, 056 (2008) [arXiv:0805.2764 [hep-th]].

[10] Zinoviev, Yu. M.: Nucl. Phys. B 785, 98 (2007) [arXiv:0704.1535 [hep-th]], Class. Quant. Grav. 26, 035022 (2009) [arXiv:0805.2226 [hep-th]], JHEP 0904, 035 (2009) [arXiv:0903.0262 [hep-th]], [arXiv:1007.0158].

[11] Manvelyan, R., Mkrtchyan, K., Ruehl, W.: Nucl. Phys. B 836, 204 (2010) [arXiv:1003.2877], [arXiv:1002.1358], Nucl. Phys. B 826, 1 (2010) [arXiv:0903.0243 [hep-th]]. Manvelyan, R., Mkrtchyan, K.: Mod. Phys. Lett. A 25, 1333 (2010) [arXiv:0903.0058 [hep-th]].

[12] Bastianelli, F., Bonezzi, R.: JHEP 0903, 063 (2009) [arXiv:0901.2311 [hep-th]]. Bastianelli, F., Corradini, O., Latini, E.: JHEP 0811, 054 (2008) [arXiv:0810.0188 [hep-th]]. Corradini, O.: [arXiv:1006.4452], Deser, S, Waldron, A.: Nucl. Phys. B 607, 577 (2001) [arXiv:hep-th/0103198]. Gover, A. R., Shaukat, A., Waldron, A.: arXiv:0812.3364 [hep-th]. Cherney, D., Latini, E., Waldron, A.: Phys. Lett. B 682, 472 (2010) [arXiv:0909.4578 
[Unknown]]. Marnelius, R.: [arXiv:0906.2084 [hep-th]].

[13] Sagnotti, A., Tsulaia, M.: Nucl. Phys. B 682 (2004) 83 [arXiv:hep-th/0311257].

[14] Buchbinder, I. L., Fotopoulos, A., Petkou, A. C., Tsulaia, M.: Phys. Rev. D $\mathbf{7 4}$ (2006) 105018 [arXiv:hep-th/0609082].

[15] Fotopoulos, A., Tsulaia, M.: Phys. Rev. D 76, 025014 (2007) [arXiv:0705.2939 [hep-th]].

[16] Fotopoulos, A., Irges, N., Petkou, A. C., Tsulaia, M.: JHEP 0710 (2007) 021 [arXiv:0708.1399 [hep-th]].

[17] Fotopoulos, A., Tsulaia, M.: JHEP 0910, 050 (2009) [arXiv:0907.4061 [hep-th]].

[18] Francia, D., Mourad, J., Sagnotti, A.: Nucl. Phys. B 773, 203 (2007)

[arXiv:hep-th/0701163]. Nucl. Phys. B 804, 383 (2008) [arXiv:0803.3832 [hep-th]]. Sagnotti, A., [arXiv:1002.3388].

[19] Bekaert, X., Joung, E., Mourad, J., JHEP 0905, 126 (2009) [arXiv:0903.3338 [hep-th]].
[20] Francia, D., Sagnotti, A.: Class. Quant. Grav. 20 (2003) S473 [arXiv:hep-th/0212185]. Francia, D.: Phys. Lett. B 690, 90 (2010) [arXiv:1001.5003].

[21] Sagnotti, A., Taronna, M.: [arXiv:1006.5242], Taronna, M.: [arXiv:1005.3061].

[22] Polyakov, D.: [arXiv:1005.5512], [arXiv:0910.5338].

[23] Giombi, S., Yin, X.: [arXiv:0912.3462], [arXiv:1004.3736].

Angelos Fotopoulos

E-mail: foto@to.infn.it

Department of Theoretical Physics

Turin University

Sezione di Torino, via P. Giuria 1, I-10125 Torino, Italy

Mirian Tsulaia

E-mail: tsulaia@liv.ac.uk

Department of Mathematical Sciences

University of Liverpool

Liverpool, L69 7ZL, United Kingdom 